MAGNETOHYDRODYNAMICS Vol. 54 (2018), No. 1-2, pp. 61-64

DOI: $10.22364 / \mathrm{mhd} .54 .1-2.11$

\title{
THE INFLUENCE OF UNIFORM EXTERNAL MAGNETIC FIELD ON HEAT TRANSFER IN FERROFLUIDS
}

\author{
M.T.Krauzina ${ }^{1}$, A.A.Bozhko ${ }^{1}$, P.V. Krauzin ${ }^{1}$, S.A.Suslov ${ }^{2}$ \\ 1 Perm State University, 15 Bukireva str., Perm 614990, Russia \\ ${ }^{2}$ Swinburne University of Technology, Hawthorn, Victoria 3122, Australia
}

Our experimental study has shown that whereas the application of a uniform magnetic field can significantly intensify heat transfer in ferrofluids, in general, it is a nonmonotonic function of the strength of the applied magnetic field.

Introduction. A spherical cavity was used to study the influence of a uniform external magnetic field on the stability of mechanical equilibrium and heat transfer in ferrofluid (FF). If the cavity is isothermal, the magnetic field inside it remains uniform [1]. Ferrocolloids based on transformer oil (TOFF) and kerosene (KFF) with magnetite particles stabilized by oleic acid were used in experiments. The nanoparticles had an average size of $10 \mathrm{~nm}$. The used FFs had, respectively, saturation magnetizations of 44.9 and $48.7 \mathrm{kA} / \mathrm{m}$, the densities $1.37 \times 10^{3}$ and $1.25 \times 10^{3} \mathrm{~kg} / \mathrm{m}^{3}$, the dynamic viscosities 0.069 and $0.009 \mathrm{~Pa} \cdot \mathrm{s}$ and volumetric concentrations of particles of 10 and $11 \%$.

In order to investigate thermomagnetic effects in non-isothermal magnetic fluids, it is necessary to create experimental conditions, where the values of magnetic and gravitational Rayleigh numbers are of the same order [2]. When a uniform external magnetic field is applied, the ponderomotive Kelvin force responsible for magnetoconvection arises due to the gradient of an internal magnetic field induced by the thermal variation of the fluid's magnetization $M$. The latter is characterized by the pyromagnetic coefficient $K=-(\partial M / \partial T)_{H}$. The expression for the magnetic Rayleigh number representing the strength of the Kelvin force relative to viscous forces is

$$
\mathrm{Rm}=\frac{\mu_{0}(K \Delta T d)^{2}}{\eta \lambda(1+\chi)}
$$

where $\mu_{0}=4 \pi \times 10^{-7} \mathrm{H} / \mathrm{m}$ is the magnetic constant, $\eta, \lambda$ and $\chi$ are the coefficients of dynamic viscosity, thermal diffusivity and magnetic susceptibility, respectively, and $\Delta T$ is the temperature difference between the poles of a sphere of diameter $d$. Thus, to achieve large values of $\mathrm{Rm}$ in a uniform external field, large temperature differences need to be applied to fluids with large pyromagnetic coefficients.

Under such conditions, thermomagnetic convection can be induced by applying a uniform vertical magnetic field even if the fluid is heated from above and remains in a stable mechanical equilibrium with respect to gravitational buoyancy [3]. The thermomagnetic parameter characterizing the dependence of the magnetic Rayleigh number on the magnetic field for a given type of $\mathrm{FF}$ is $K^{2} /(1+\chi)$, which was described in detail in the case of a flat fluid layer in [4]. To compare with the present experimental results more straightforwardly, the dependence of this parameter on the strength $H$ of the applied magnetic field that takes into account its reduction inside a spherical cavity filled with a magnetic fluid [1] is shown in Fig. 1a. It is conventional to use the Langevin law to describe the magnetization of FFs [5], which is a reasonable approximation for fluids with 
$(a)$

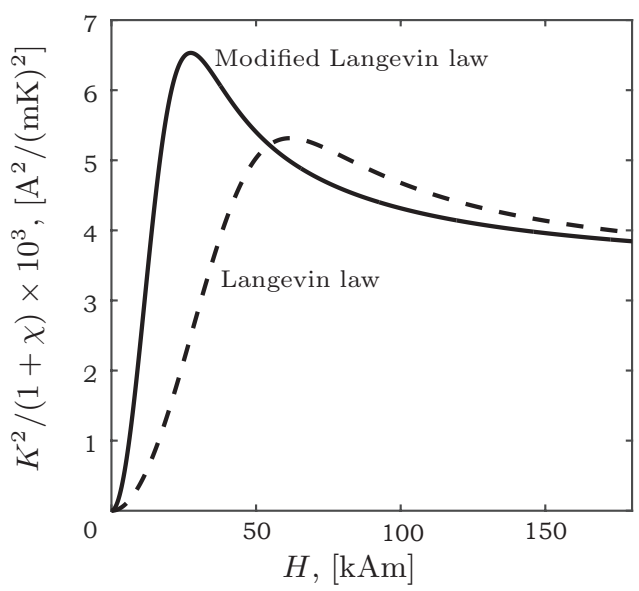

(b)

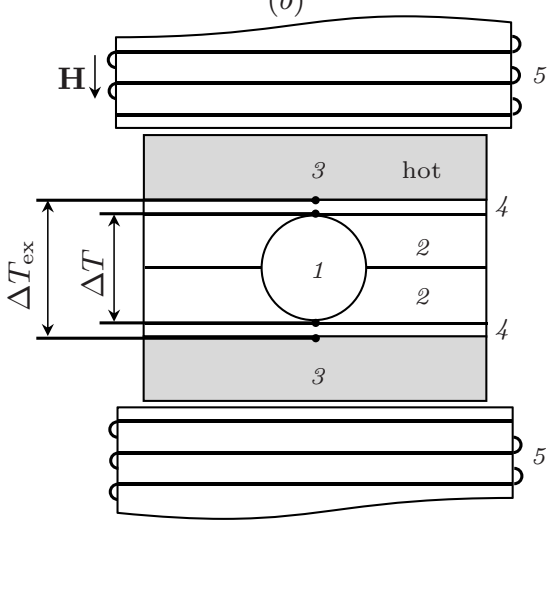

Fig. 1. (a) Variation of the parameter characterizing thermomagnetic heat transfer in KFF (with the initial magnetic susceptibility $\chi=2.88$ ) as a function of the applied magnetic field; (b) schematic presentation of the experimental chamber: 1 - spherical cavity filled with magnetic fluid, 2-Plexiglas plates, 3-aluminium heat exchangers, 4 - Plexiglas inserts, 5 - electromagnets.

a small concentration of the magnetic phase [6]. Yet, for more concentrated fluids, the Langevin law becomes rather inaccurate, since it does not consider the interaction between magnetic particles. Thus, the second-order modified Langevin law based on the mean field approximation, which is in a better agreement with experimental field measurements for concentrated FFs, has been suggested in [7]. The results computed using both laws are presented for our experimental fluids in Fig. 1a. Despite that there is a noticeable quantitative difference between the two sets of results, both magnetization laws demonstrate the existence of the maximum of the magnetization parameter and, hence, of the maximum achievable value of the magnetic Rayleigh number. From the experimental point of view, this means that increasing the value of $\mathrm{Rm}$ by increasing the applied field can only be done for $H \lesssim 30 \mathrm{kA} / \mathrm{m}$. The value of Rm for stronger fields decreases before levelling out when the fluid approaches magnetic saturation.

1. Experimental setup. The schematic view of the experimental chamber is shown in Fig $1 b$. A spherical cavity 1 of the diameter $d=16.0 \pm 0.1 \mathrm{~mm}$ was formed by two hemispheres carved in the Plexiglas plates 2 and glued together along the equatorial plane. The overall dimensions of the Plexiglas block were $53 \times 53 \times 18 \mathrm{~mm}^{3}$. The block was placed between two aluminium heat exchangers 3 . The temperatures of the heat exchangers were controlled by jet ultra-thermostats that maintained the temperature of the water within $\pm 0.02 \mathrm{~K}$ of the set value that was pumped through the channels drilled in the aluminium plates. The uniform external magnetic field (up to $200 \mathrm{kA} / \mathrm{m}$ ) was created by Helmholtz coils or by an electromagnet.

Since the working fluids were not transparent, optical methods of observations were not possible. Instead the temperature measurements were made for detecting and quantifying the strength of convection flows. In a spherical cavity they take the form of a vortex with the axis located in the equatorial plane of the cavity $[3,8]$. When they arise, the symmetry of the temperature distribution breaks, and this fact can be used to detect the onset of convection. In our experiments, the temperature differences $\Delta T$ and $\Delta T_{\text {ex }}$ between the poles of the sphere and 
The influence of uniform external magnetic field on heat transfer in ferrofluids

(a)

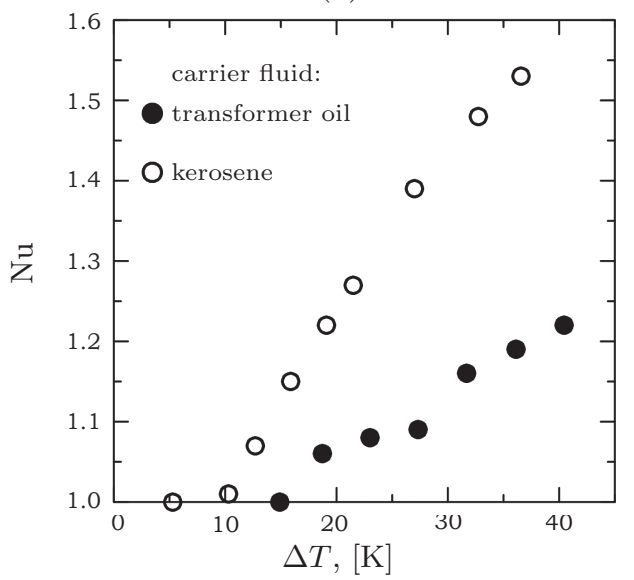

(b)

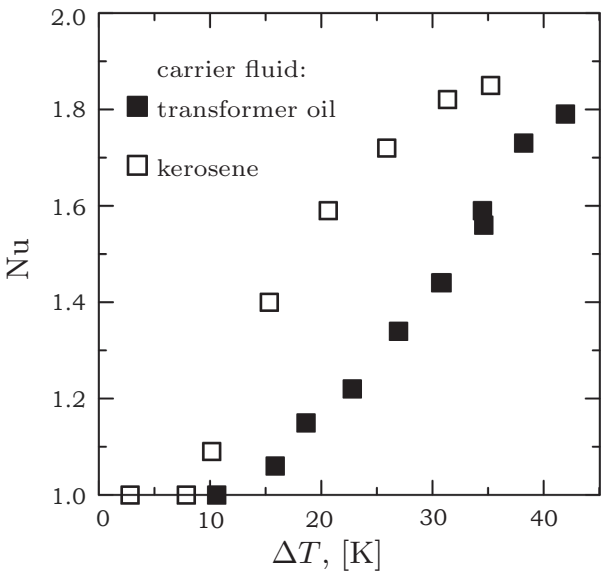

Fig. 2. Dimensionless heat flux through a spherical cavity filled with FF heated from above and exposed to the uniform vertical magnetic field with intensity (a) $15.9 \mathrm{kA} / \mathrm{m}$ and (b) $30 \mathrm{kA} / \mathrm{m}$.

the heat exchangers, respectively, were registered using copper-constantan thermocouples located along the $1 \mathrm{~mm}$ thick Plexiglas inserts 4. The coefficient of the thermocouples' thermo-e.m.f was $40 \mu \mathrm{V} / \mathrm{K}$, the electrodes diameter was $0.1 \mathrm{~mm}$ and the length of the soldered junction was $1 \mathrm{~mm}$.

The intensity of the vertical heat transfer across the cavity heated from above and exposed to the magnetic field was characterized by the Nusselt number that is the ratio of the full heat flux to its conduction component. It was determined experimentally as $\mathrm{Nu}=\Delta T_{\mathrm{s}} /(k \Delta T)$, where $\Delta T_{\mathrm{s}}=\Delta T_{\mathrm{ex}}-\Delta T$ is the temperature drop across the inserts and $k$ is an empirical constant representing the ratio of the heat conductivities of the fluid and Plexiglas.

2. Results. As follows from Fig. 2, thermomagnetic convection sets in the $\mathrm{KFF}$ at smaller temperature differences than in the TOFF. Partially, this is due to the smaller viscosity of kerosene. The heat flux in the KFF is larger than in the TOFF at all temperature differences. For example, as seen from Fig. 2, the heat transfer in the KFF at $\Delta T \approx 35 \mathrm{~K}$ in the magnetic field $H=15.9 \mathrm{kA} / \mathrm{m}$ is $27 \%$, and at $H \approx 30 \mathrm{kA} / \mathrm{m}$ it is by $16 \%$ stronger than in the TOFF. The comparison of Figs. $2 a$ and $2 b$ shows that the heat transfer intensifies when the applied magnetic field and, hence, the fluid magnetization increase. This is due to the strengthening of the Kelvin force characterized by the increase of the magnetic Rayleigh number (see Fig. 1a).

Fig. $3 a$ demonstrates that the heat flux in the TOFF increases as the magnetic field strengthens and reaches the maximum at $H>60 \mathrm{kA} / \mathrm{m}$. The heat flux in the KFF also grows initially and reaches the maximum $\mathrm{Nu}=2.3$ at $H=23.9 \mathrm{kA} / \mathrm{m}$. However, subsequently, the heat flux drops sharply to $\mathrm{Nu}=1.1$ at $H=106.6 \mathrm{kA} / \mathrm{m}$, which is close to the value measured in weak fields. In magnetic fields that are stronger than $25 \mathrm{kA} / \mathrm{m}$, where the value of the thermomagnetic parameter starts decreasing from its maximum (see Fig. 1a), the stabilizing factors, such as the fluid density stratification (arising due to the gravitational sedimentation of solid particles and their aggregates) and the magnetoviscous effect become dominant. A similar transition from a promoting to a suppressing action of the increasing magnetic field on convection flows was previously observed in experiments with a flat FF layer [2]. 
M.T. Krauzina, A.A.Bozhko, P.V.Krauzin, S.A.Suslov

(a)

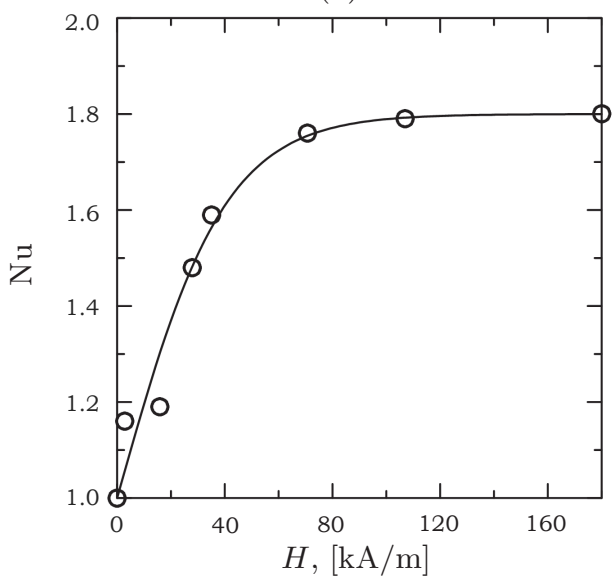

(b)

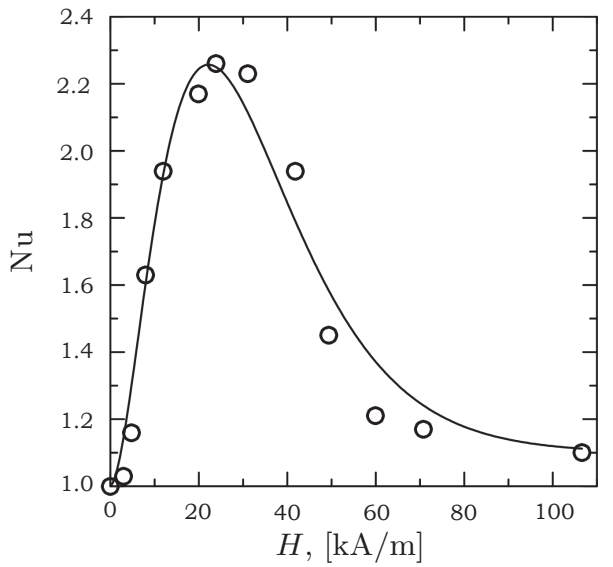

Fig. 3. The Nusselt number as a function of the magnetic field intensity (heating from above): (a) TOFF, $\Delta T_{\mathrm{ex}}=41.8 \mathrm{~K}$, and (b) KFF, $\Delta T_{\mathrm{ex}}=45.0 \mathrm{~K}$.

3. Conclusions. The application of a uniform external magnetic field can lead to a significant (up to 100\%) increase of the heat flux in KFFs and TOFFs due to the thermomagnetic convective transfer. However, whereas the monotonic intensification of heat transfer and its subsequent saturation are observed in increasing magnetic fields in the TOFF, in the KFF the initial increase of the heat transfer intensity is followed by its sharp decrease in stronger fields.

Acknowledgements. MTK and PVK acknowledge financial support by the Russian Foundation for Basic Research (grant no. 16-31-00091 mol_a).

\section{References}

[1] C. Kalashnikov. Electricity (Nauka, Moscow, USSR, 1977).

[2] A.A. Bozhko, G.F. Putin. Heat transfer and flow patterns in ferrofluid convection. Magnetohydrodynamics, vol. 39 (2003), no. 2, pp. 147-169.

[3] M.T. Krauzina, A.A. Bozhko, P.V. Krauzin, and S.A. Suslov. Oscillatory instability of convection in ferromagnetic nanofluid and in transformer oil. Fluid Dyn. Res., vol. 48 (2016), no. 6, 061407.

[4] H. Rahman and S.A. Suslov. Thermomagnetic convection in a layer of ferrofluid placed in a uniform oblique external magnetic field. J. Fluid Mech., vol. 764 (2015), pp. 316-348.

[5] R.E. Rosensweig. Ferrohydrodynamics (Cambridge University Press, 1985).

[6] S.V.D'yachenko And A.I.Zhernovor. The langevin formula for describing the magnetization curve of a magnetic liquid. Tech. Phys., vol. 61 (2016), no. 12 , pp. $1835-1837$.

[7] A.O. Ivanov, et al. Magnetic properties of polydisperse ferrofluids: A critical comparison between experiment, theory, and computer simulation. Phys. Rev. E, vol. 75 (2007), p. 061405.

[8] A.P. Ovchinnikov and G.F. Shaidurov. Convective stability of homogeneous fluid in a spherical cavity. Hydrodynamics. Perm State University, vol. 1 (1968), pp. 3-21 (in Russian). 